\title{
Modeling, Identification, and Control of a Pneumatically Actuated, Force Controllable Robot
}

\author{
James E. Bobrow and Brian W. McDonell
}

\begin{abstract}
This research focuses on modeling and control of a light-weight and inexpensive pneumatic robot that can be used for position tracking and for end-effector force control. Unlike many previous controllers, our approach more fully accounts for the nonlinear dynamic properties of pneumatic systems such as servovalve flow characteristics and the thermodynamic properties of air compressed in a cylinder. We show with theory and experiments that pneumatic actuators can rival the performance of more common electric actuators. Our pneumatic robot is controlled by extending existing manipulator control algorithms to handle the nonlinear flow and compressibility of air. The control approach uses the triangular form of the coupled rigid body and air flow dynamics to establish path tracking. In addition to the trajectory tracking control law, a hybrid position/force control algorithm is developed. The experimental results indicate that the tip forces on the robot can be controlled without the need for an expensive force/torque sensor usually required by electric motor driven systems.
\end{abstract}

Index Terms - Force control, hierarchical control, modeling, nonlinear control, pneumatic control systems.

\section{INTRODUCTION}

W HERE originally nearly all controlled motion was done using electric motors, as computers have become more powerful, other forms of actuation have become feasible for providing motion. In addition, as the complexity of robotic tasks has increased, position control alone has not been sufficient. Useful interaction with the environment requires producing controlled forces in addition to moving the robot with a prescribed motion. Pneumatic actuation, originally discounted as not being suitable for anything other than motion between two hard stops, is now becoming a reasonable substitute for electric actuation in some applications. Pneumatic actuators are of interest for robotic applications because of their large power output at a relatively low cost. They are also clean, easy to work with, and lightweight. In addition, compressed air is readily available at nearly every industrial facility. Unfortunately, position stabilization of a pneumatic actuator is difficult if a high bandwidth closed-loop system is desired.

Manuscript received September 4, 1997; revised July 13, 1998. This work was supported by the Rockwell International Graduate Fellowship Program, by NSF Grant MSS-9006667, and by Parker-Hannifin, Parker-Bertea Aerospace Division. This paper was recommended for publication by Associate Editor K. Tanie and Editor A. De Luca upon evaluation of the reviewers' comments.

J. E. Bobrow is with the Department of Mechanical and Aerospace Engineering, University of California, Irvine, CA 92697 USA.

B. W. McDonell is with ATL Products, Irvine, CA 92614 USA.

Publisher Item Identifier S 1042-296X(98)07435-7.
Several controllers have been used in the past for pneumatic systems. Most have been fixed gain linear controllers based on a nominal transfer function model obtained by linearizing the air flow dynamics about the cylinder midstroke position [1]-[3]. Later work by Liu and Bobrow [4] has used a linearized state space model to develop an optimal regulator for a fixed operating point. In order to deal with the uncertainties and the highly nonlinear behavior of pneumatic systems, a number of approaches have also been developed that incorporate some form of learning. Pu and Weston [5] describe an algorithm which is trained to provide feed-forward signals to optimize various point to point motions. McDonell and Bobrow [6] use a real time identification scheme to identify a locally linear time-varying model for the system about arbitrary reference trajectories.

In the pursuit of better performance in pneumatic systems for less cost, research has been done on different hardware configurations. One component that is costly and difficult to model for pneumatic controllers is the servovalve. A possible substitute is to replace the variable flow servovalve with a linear or rotary solenoid valve. Using a solenoid valve, a pulse-width modulated control law that has a fixed-gain inner pressure loop and an outer position loop was developed in Lai et al. [7]. Other controllers using solenoid valves based on sliding mode control theory have been developed by Tang and Walker [8] and by Paul et al. [9]. A reduced order sliding surface was used for the second approach to eliminate the need for pressure feedback. A novel rotary type air flow control valve and its control system has been developed by Kunt and Singh [10]. In this work, a pulse-width modulated form of control is applied to a linear time-varying system model with good results.

More recently, nonlinear control laws have been developed using the full nonlinear dynamics of pneumatic systems using feedback linearization techniques [11], [12], or Lyapunov stability arguments [13]. When compared to control laws based on linearization, the nonlinear control laws obtained with these approaches dramatically extend the range of stability. The basic form of the control law obtained in the above references is similar to that obtained in our research. That is, they all require a model of the air flow dynamics, and they all use air pressure feedback in a level of control that drives the pressure dynamics to a desired output. As suggested by Kawamura et al. [13], we develop a natural separation between the rigid body dynamics and the actuator dynamics in a hierarchical 
control approach. We focus on obtaining an accurate air flow model that can be inverted to implement any of these nonlinear control approaches.

Although previous applications of pneumatic actuators to robotics have primarily used linearized controllers, their low cost, light weight, and ability for output force control makes them attractive alternatives to electric motors. For instance, an important early use of pneumatics was for tendon actuation of the Utah/MIT dexterous hand [14]. Later work on accurate force control of these tendons was conducted by Henri and Hollerbach [15] using a linearized model of air flow through a jet-pipe. Ben-Dov and Salcudean [16] also developed a force controllable pneumatic system with low friction cylinders that can be used for robotic teleoperation applications. Their controller is based on a linearized model of flow through a flapper valve. Another application of pneumatics is a three degree of freedom wrist and linear controller presented in Pfruendschuh et al. [17]. This system uses the compressibility of air to obtain natural compliance on a parallel tripod platform. Chirikjian [18] has also exploited the low cost feature of pneumatic systems in the development of "binary manipulators" which use a large number of pneumatic actuators in a state of full extension or full contraction.

Our work has the advantages of the above mentioned servovalve actuated pneumatic systems and as mentioned earlier, is not limited by the choice of operating point as are the controllers based on linearization. In the following, we first derive a nonlinear dynamic model of the actuator dynamics and then demonstrate with experimental evidence that common assumptions made for the valve flow dynamics are not accurate. Next we combine the actuator dynamics with the nonlinear rigid body dynamics of a $3 \mathrm{R}$ robot to obtain a model of the coupled system. We then develop a model based controller that provides exponentially stable path tracking and output force control for the coupled system using the full nonlinear dynamics. Finally experiments are presented that demonstrate the strengths of our approach and its limitations due to realistic considerations such as friction and valve flow saturation.

\section{ROBOT DESCRIPTION}

The experimental $3 \mathrm{R}$ robot used in this research is shown in five different positions in Fig. 5. Fig. 1 shows the basic configuration of each of the three actuators used. Each actuator consists of a double acting pneumatic cylinder driving, via cables, a rotary joint. A control current is sent to a servovalve to control the spool position that creates an air flow into or out of the two cylinder chambers.

Joint 1 of the robot is attached to the robot base and creates a rotation out of plane for the shoulder-elbow configuration of joints 2 and 3 which are both 18 in $(45.7 \mathrm{~cm})$ in length. A unique feature of the robot is that the structural portion of each link is also the pneumatic cylinder that provides rotary actuation for the subsequent link. That is, the cable in Fig. 1 drives the right-hand pulley, and this pulley is attached to the next cylinder in the kinematic chain. Each cylinder has a bore of 3 in $(7.6 \mathrm{~cm})$ and is operated at a supply pressure of 100 psig $(689 \mathrm{kPa})$ which gives a maximum linear force output on

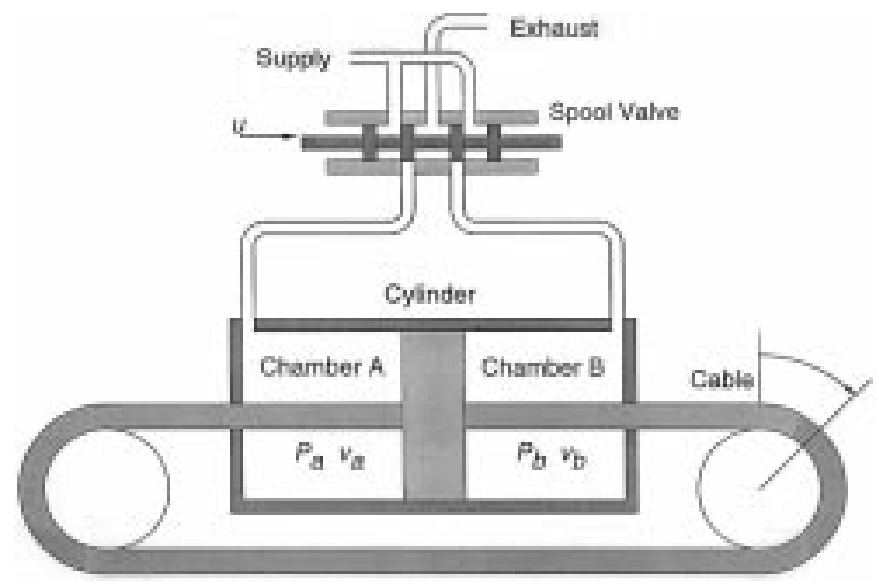

Fig. 1. Schematic diagram of a pneumatic actuator.

each cable of approximately $700 \mathrm{lb}(3100 \mathrm{~N})$. The radius of each pulley was chosen to give the robot a maximum payload capacity of $20 \mathrm{lb}(89 \mathrm{~N})$ in the fully outstretched configuration. The total weight of the robot, including the valves and base is approximately $50 \mathrm{lb}(220 \mathrm{~N})$. An interesting property of this pneumatic robot is that it can hold an object in the outstretched position indefinitely with no heating or power loss. This is because once the correct mass of air has entered the cylinders to balance the load, no further air flow or valve control signal is needed.

The robot was controlled using a 486 PC. The feedback sensors for each joint included a piezoresistive pressure transducer on each side of the piston (two sensors needed on each joint) and a 432000 pulse/revolution position encoder. The control signal was a voltage obtained from the PC through a 12 bit D/A converter. This voltage was converted to a valve current using an analog voltage to current loop. The servovalve was a standard jet-pipe type valve [19] with a maximum flow rate of $10 \mathrm{cfm}$ (283 liter/min) at a pressure drop of $100 \mathrm{psi}$ (689 $\mathrm{kPa}$ ) across the valve. Although the supply pressure was regulated to $100 \mathrm{psig}$, a pressure transducer was also used in the feedback control loop that measured the instantaneous supply pressure near the inlet to the servovalves.

\section{Pneumatic Actuator Model}

A dynamic model for the behavior of air in the cylinder is developed by following Shearer [1] and Liu and Bobrow [4]. In their approach, by using conservation of energy, the relationship between mass flow rate of air and the change of pressure in chamber A (or chamber B) can be found. The internal energy of the mass flowing into the system is $c_{p} T_{s} \dot{m}_{a}$, (these variables are defined in the Appendix) the rate at which work is done on the moving piston is $p_{a} \dot{v}_{a}$, the rate of heat transfer through the wall is $\dot{Q}$, and the rate of change of the internal energy of the air in the cylinder is $\left(c_{v} / R\right)(d / d t)\left(p_{a} v_{a}\right)$. Here $\dot{m}_{a}$ is the mass flow rate of air to chamber $\mathrm{A}, c_{v}$ and $c_{p}$ are the constant volume and constant pressure specific heats of air, $R$ is the universal gas constant, $v_{a}$ is the volume of chamber $\mathrm{A}, T_{s}$ is the air supply temperature, and $p_{a}$ is the cylinder pressure. Summing these 
terms in an energy balance yields

$$
c_{p} T_{s} \dot{m}_{a}-p_{a} \dot{v}_{a}+\dot{Q}=\frac{c_{v}}{R} \frac{d}{d t}\left(p_{a} v_{a}\right) .
$$

For this actuator shown in Fig. 1, the cylinder volumes are related to the pulley position by

$$
\begin{aligned}
& v_{a}=A_{c} r \theta+v_{a, \text { end }} \\
& v_{b}=A_{c}(l-r \theta)+v_{b, \text { end }}
\end{aligned}
$$

where $A_{c}$ is the cylinder cross-sectional area, $l$ is the stroke, $r$ is the radius of the pulley that rotates the pulley, $v_{a, \text { end }}$ and $v_{b, \text { end }}$ are the fixed volumes at the ends of the stroke, and $\theta$ is the angle of rotation of the pulley with $\theta=0$ when the piston is at the left end of the cylinder.

The rate of heat transfer $\dot{Q}$ is governed by the temperature difference between the inside and outside of the cylinder and the coefficients of thermal conductivity of the stainless steel cylinder and the aluminum end-caps. For the experimental system the thermal time constant for this heat transfer process is on the order of several minutes, which was assumed to be much slower than the response time of the robot. We therefore assume that $\dot{Q}=0$. Experimental studies related to the validity of this assumption have been reported in [20]. With $\dot{Q}=0$, expand (1), and use the fact that $R=c_{p}-c_{v}$, to obtain

$$
\begin{aligned}
p_{a} \dot{v}_{a}+\dot{p}_{a} v_{a} & =R \frac{c_{p}}{c_{v}} T_{s} \dot{m}_{a}-\frac{R}{c_{v}} p_{a} \dot{v}_{a} \\
\dot{p}_{a} v_{a} & =R \frac{c_{p}}{c_{v}} T_{s} \dot{m}_{a}-\frac{R+c_{v}}{c_{v}} p_{a} \dot{v}_{a} \\
\dot{p}_{a} & =R \frac{c_{p}}{c_{v}} T_{s} \frac{\dot{m}_{a}}{v_{a}}-\frac{c_{p}}{c_{v}} \frac{p_{a} \dot{v}_{a}}{v_{a}} .
\end{aligned}
$$

Similarly for the $b$ side of the cylinder

$$
\dot{p}_{b}=R \frac{c_{p}}{c_{v}} T_{s} \frac{\dot{m}_{b}}{v_{b}}-\frac{c_{p}}{c_{v}} \frac{p_{b} \dot{v}_{b}}{v_{b}} .
$$

The mass flow rates, $\dot{m}_{a}$ and $\dot{m}_{b}$ are nonlinear functions of the servovalve spool position $u$ and the supply and cylinder pressures. In addition, the servovalve has dynamics relating the control current to spool position which have a $-3 \mathrm{~dB}$ amplitude ratio and a $90^{\circ}$ phase lag at 40 hertz as described in the manufacturers literature [19]. To simplify the model, the spool valve position is assumed to be directly proportional to the control current. The dynamics of the servovalve can be included in the formulation if one extends the levels of control hierarchy described in Section V-B to three.

The torque acting on the joint is related to the difference in pressure between the two sides of the cylinder, so that $\tau=A_{c} r\left(p_{a}-p_{b}\right)$, or

$$
\dot{\tau}=A_{c} r\left(\dot{p}_{a}-\dot{p}_{b}\right) .
$$

Substituting (6) and (7) into (8) and separating $\dot{\tau}$ into the terms affected by the servovalve spool position $u$ and the terms which are functions only of the position and velocity of the joint we obtain

$$
\dot{\tau}=A_{c} r R \frac{c_{p}}{c_{v}} T_{s}\left(\frac{\dot{m}_{a}}{v_{a}}-\frac{\dot{m}_{b}}{v_{b}}\right)-A_{c} r \frac{c_{p}}{c_{v}}\left(\frac{p_{a} \dot{v}_{a}}{v_{a}}-\frac{p_{b} \dot{v}_{b}}{v_{b}}\right)
$$

or

$$
\dot{\tau}=\hat{u}\left(u, p_{a}, p_{b}, v_{a}, v_{b}\right)+\hat{h}\left(p_{a}, p_{b}, v_{a}, v_{b}, \dot{v}_{a}, \dot{v}_{b}\right)
$$

where

$$
\begin{aligned}
\hat{u}\left(u, p_{a}, p_{b}, v_{a}, v_{b}\right)= & A_{c} r R \frac{c_{p}}{c_{v}} T_{s} \\
& \cdot\left(\frac{\dot{m}_{a}\left(u, p_{a}\right)}{v_{a}}-\frac{\dot{m}_{b}\left(u, p_{b}\right)}{v_{b}}\right) \\
\hat{h}\left(p_{a}, p_{b}, v_{a}, v_{b}, \dot{v}_{a}, \dot{v}_{b}\right)= & -A_{c} r \frac{c_{p}}{c_{v}}\left(\frac{p_{a} \dot{v}_{a}}{v_{a}}-\frac{p_{b} \dot{v}_{b}}{v_{b}}\right)
\end{aligned}
$$

and we have assumed that $\dot{m}_{a}$ and $\dot{m}_{b}$ are functions of $u$ and the cylinder pressures.

To more easily represent multiple degree of freedom pneumatic systems, these equations will now be considered $n$ vectors with each element corresponding to each independent joint and actuator, i.e.,

$$
\dot{\tau} \equiv\left\{\begin{array}{c}
\dot{\tau}_{1} \\
\vdots \\
\dot{\tau}_{n}
\end{array}\right\}=\hat{u}+\hat{h} \equiv\left\{\begin{array}{c}
\hat{u}_{1} \\
\vdots \\
\hat{u}_{n}
\end{array}\right\}+\left\{\begin{array}{c}
\hat{h}_{1} \\
\vdots \\
\hat{h}_{n}
\end{array}\right\} .
$$

\section{VAlve Flow Characterization}

The control law developed in the following section requires knowledge of all of the parameters and states of the air flow dynamics in (9). Most of the parameters used in (9) are easily measured such as the cylinder area and the pulley radius. The most difficult quantities to characterize are the mass flow rates through the valve. For the purpose of modeling, it is often assumed that the mass flow of air through the servovalve closely matches that of airflow through an orifice mounted in a pipe where the orifice area varies with control current. We will call this the "theoretical" flow rate in what follows. The equations governing mass flow through an orifice with no losses are given in [21] (14), shown at the bottom of the page, where $A_{0}$ is the effective valve orifice area, $p_{1}$ is the upstream pressure, $p_{2}$ is the downstream pressure, $T_{1}$ is the upstream temperature, and $k=c_{p} / c_{v}=1.4$ for air.

For the pneumatic system there are two situations which are encountered:

1) a constant pressure supply flowing into the cylinder;

2) the cylinder exhausting into atmospheric pressure.

$$
\dot{m}=\left\{\begin{array}{l}
\frac{A_{o}}{\sqrt{T_{1}}} \sqrt{\frac{k}{R} \frac{2}{k-1}} p_{1} \sqrt{\left(\frac{p_{2}}{p_{1}}\right)^{2 / k}-\left(\frac{p_{2}}{p_{1}}\right)^{k+1 / k}}: \frac{p_{2}}{p_{1}}>\left(\frac{2}{k+1}\right)^{k / k-1} \quad \text { (unchoked) } \\
\frac{A_{o}}{\sqrt{T_{1}}} \sqrt{\frac{k}{R}\left(\frac{2}{k+1}\right)^{k+1 / k-1}} p_{1}: \frac{p_{2}}{p 1} \leq\left(\frac{2}{k+1}\right)^{k / k-1} \quad \text { (choked) }
\end{array}\right.
$$




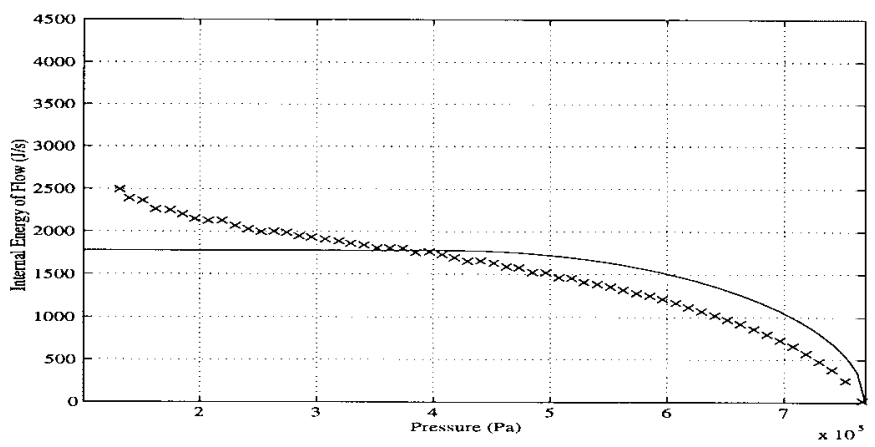

Fig. 2. Curve fit for $c_{p} T_{s} \dot{m}_{a}$ as a function of $p_{a}$ with a valve control current of $6 \mathrm{~mA}$

In both the filling and exhausting situations it is possible to reach conditions of choked flow. We tested the validity of (14) using the following experimental procedure. First note that (6) shows that for a fixed cylinder volume $v_{a}$ and constant $T_{s}, \dot{m}_{a}$ is directly proportional to $\dot{p}_{a}$. We measured the internal energy of mass flowing into the system, $c_{p} T_{s} \dot{m}$ rather than $\dot{m}$ since $c_{p} T_{s} \dot{m}$ is needed for the control law. This was also done to eliminate the need for an upstream temperature sensor on the assumption that in terms of absolute temperatures, the supply temperature will be changing very little. With $u=0$ chamber A was moved to its maximum volume configuration and exposed to atmospheric pressure. The chamber was then closed and a constant control $u$ was sent to the servovalve. For this constant $u(t)$, the pressure $p_{a}(t)$ and the supply pressure $p_{\text {sup }}(t)$ were recorded at $200 \mathrm{~Hz}$ as the cylinder filled with air. Given the measured $p_{a}(t), p_{\mathrm{sup}}(t)$, we then used a central difference numerical derivative to obtain the corresponding $\dot{p}_{a}(t)$. In this manner, $c_{p} T_{s} \dot{m}_{a}$ is also known from (6). Note that although the supply pressure is usually assumed to be constant, we found it better to measure the actual upstream pressure near the valve. The upstream pressure did fluctuate during period of high flow transients.

Given the above data, the only unknown in (14) is the effective orifice area $A_{o}(u)$. We chose the unknown constant $A_{o}$ that produced the least mean-squared error from our measured data and the theoretical curve given by (14). A typical resulting best fit solution for a given valve current $u=6 \mathrm{~mA}$ is shown in Fig. 2. The solid line is a plot of $c_{p} T_{s} \dot{m}_{a}$ versus downstream pressure from (14) with this best choice of $A_{o}(u)$, and with the upstream pressure regulated at $100 \mathrm{psig}$, or $790 \mathrm{kPa}$ absolute. The $\times$ 's denote experimentally measured data points. The discrepancy between the curves is probably due to the assumption used in the derivation of (14) that there are no losses in the flow through the orifice. Nearly all previous results on pneumatic control incorrectly assume (14) is true, or worse, assume a linear relation between $\dot{m}$ and $u$. Note that as $u$ varies, a surface of flow curves of this form are defined.

We measured a least squares surface fit by sweeping the current $u$ though its range of possible operating values and performing the above curve fitting procedure at 40 discrete values. This produced 40 effective areas for $A_{o}(u)$, which varied approximately linearly with the valve current. Because of the relatively poor match shown in Fig. 2 of the experimen-

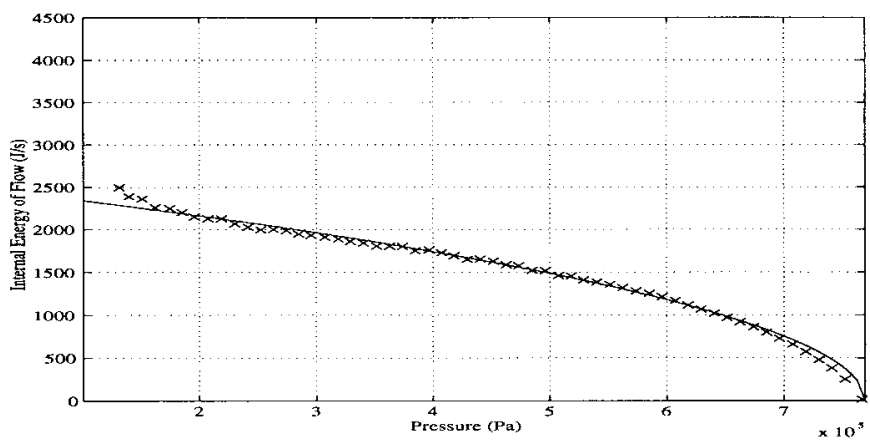

Fig. 3. New function curve fit using the same data as Fig. 2.

tally measured data to the theoretically predicted flow-rates, least squares surface fitting was done with different surfaces as basis functions. Two surfaces are needed-one that represents cylinder filling from the supply and the other that represents cylinder exhausting to atmosphere. Our control law requires that these functions of cylinder pressure and servovalve current be able to be back-solved (i.e., one to one and invertable) for the control $u$ that will produce a given $\dot{m}$ at any given cylinder pressure.

After many experiments and trials, the basis functions which yielded a good curve fit for the change in internal energy as a function of cylinder pressure were quadratic in $u$

$$
\begin{array}{ll}
c_{p} T_{s} \dot{m}=\sqrt{p_{\mathrm{sup}}-p_{a}}\left(c_{f 1} u+c_{f 2} u^{2}\right) & \text { filling } \\
c_{p} T_{s} \dot{m}=\left(p_{a}-p_{\mathrm{exh}}\right)\left(c_{e 1} u+c_{e 2} u^{2}\right) & \text { exhausting }
\end{array}
$$

where $c_{f 1}, c_{f 2}, c_{e 1}$, and $c_{e 2}$ are coefficients found from a least squares fit of the experimental data. A different set of coefficients was identified for each side of the cylinder since different valve orifices are used to produce flow in each chamber. Note that these equations use the upstream pressure. If this pressure is constant, then it need not be measured at each sample. However, as mentioned previously, we found that the upstream pressure dropped during periods of high flow, and that it is helpful to measure this pressure at each sampling instant. Fig. 3 shows the curve fit using the new function and the same data that was used in Fig. 2.

The control law in the next section sets a desired value for $\dot{\tau}$ at each sampling instant. The above flow equations must then be back-solved at each $t$ for the $u$ that produces the desired $\dot{\tau}$. This can be done since the entire state is measured, and the only unknown in (10) given $\dot{\tau}$ is $u$. In order to solve for $u$, we first determine the desired $\hat{u}$, and then insert $\dot{m}_{a}\left(u, p_{a}\right)$ and $\dot{m}_{b}\left(u, p_{b}\right)$ in (11) with the appropriate functions. If the desired $\hat{u}$ is positive, the proper value of $u$ will be one that makes chamber A fill with air and chamber B exhaust air. Therefore $\dot{m}_{a}\left(u, p_{a}\right)$ will be replaced with (15) for chamber A and $\dot{m}_{b}\left(u, p_{b}\right)$ with (16) for chamber B. If the desired $\hat{u}$ is negative, $\dot{m}_{a}\left(u, p_{a}\right)$ will be replaced with (17) and $\dot{m}_{b}\left(u, p_{b}\right)$ with (15). Following these replacements, we now have a quadratic equation which can be solved for $u$.

\section{Control Law for the Penumatic System}

As mentioned in the introduction, there are several choices possible for the control law for the pneumatic system. In 

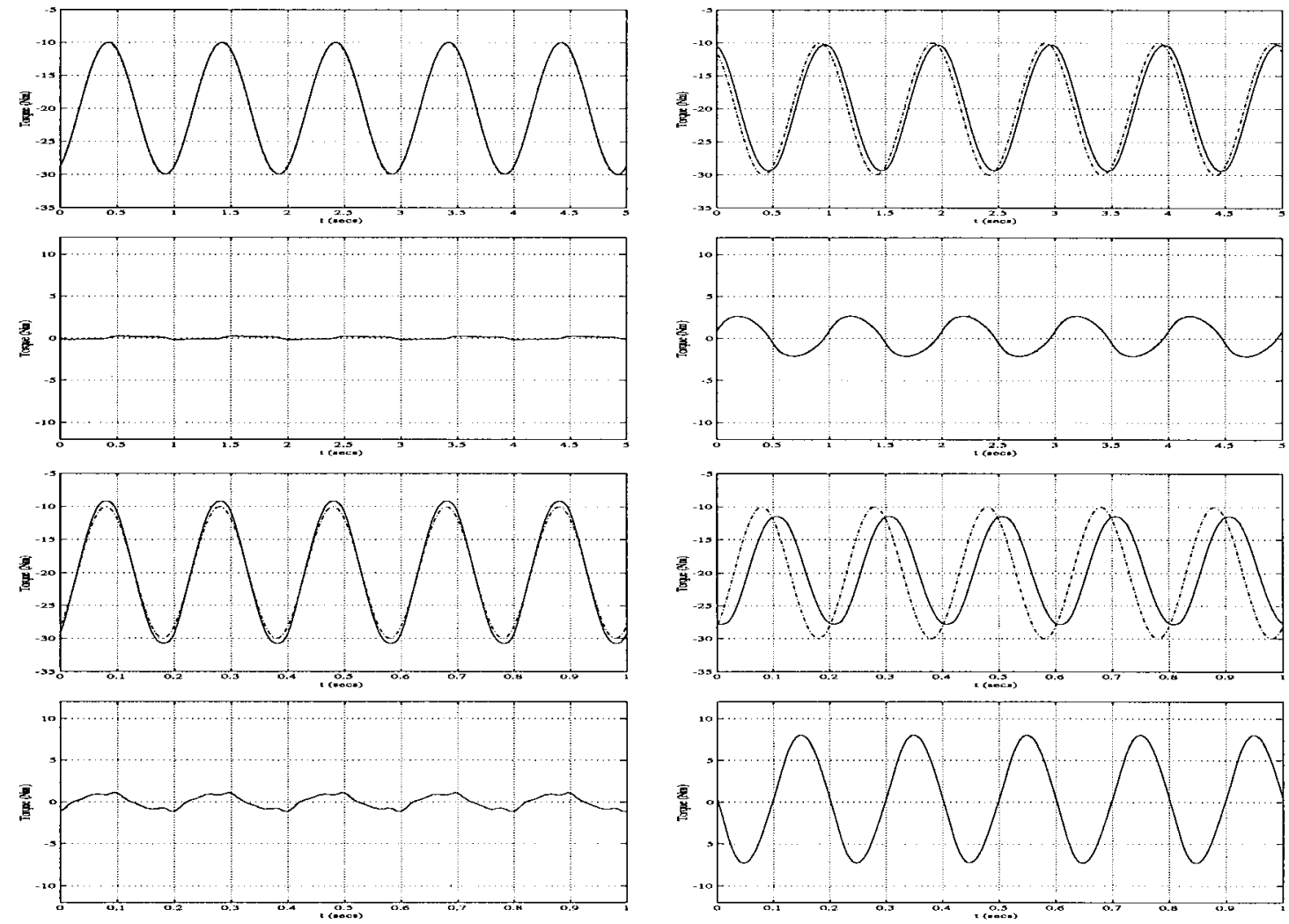

Fig. 4. The left-hand plots are 2 and $5 \mathrm{~Hz}$ sin wave tracking using the proposed control law. The tracking error is shown below them. The right-hand plots are results using the same inputs and a well-tuned proportional control law.

[22], three distinct approaches were analytically developed, and tested experimentally. Once suitable feedback gains were chosen, similar performance was achieved for all the approaches. The following control law is based on the stability of hierarchical systems and it has the advantage that it is simple to implement. The control law is also similar to integrator backstepping [23], where the control law for one subsystem is the reference signal for another. We show that any control law for standard torque controlled robots can easily be extended to pneumatic systems as long as the control law is continuously differentiable.

\section{A. Torque Control Subsystem}

The first step in the control design is to control the actuator torques. This controller will be the first subsystem in the hierarchical system. Joint torque control is necessary for any robotic output force control operation. It will also bridge the gap between the standard torque input controllers and the pneumatic robot. Consider the equation relating the valve current and the actuator torque (10), $\dot{\tau}=\hat{u}+\hat{h}$. Given any desired torque, $\tau_{s}$, and its time derivative, $\dot{\tau}_{s}$, let

$$
\hat{u}=\dot{\tau}_{s}-\hat{h}+K\left(\tau-\tau_{s}\right) .
$$

Where $K$ is a gain matrix with $K<0$.

Now, using the procedure described in the last section, solve (11) for the servovalve currents, $u$, which will produce this $\hat{u}$. This controller can easily be shown to exponentially converge to the desired torque as follows. Define the torque error as $d=\tau-\tau_{s}$ with its derivative $\dot{d}=\dot{\tau}-\dot{\tau}_{s}$. Substituting (17) into (10) and using the definition for $d$ we get

$$
\begin{aligned}
& \dot{\tau}=\dot{\tau}_{s}-\hat{h}+K\left(\tau-\tau_{s}\right)+\hat{h} \\
& \dot{d}=K d .
\end{aligned}
$$

Thus since $K<0$, the torque error, $d$, will converge to zero exponentially.

The control law (17) has a feedforward term $\dot{\tau}_{s}(t)$, a term that accounts for motion of the cylinder $\hat{h}$, and an expected proportional term. Although the proportional term $K\left(\tau-\tau_{s}\right)$ appears to be a linear control, the inversion of the flow equation (11) needed to obtain the servovalve current $u$ is highly nonlinear. Fig. 4 shows some typical experimental results for force tracking. In this case, the robot was held stationary. The left-hand plots show the response obtained with our control law for joint 1 . In the top left plot, the desired torque $\tau_{s}(t)$ is a $10 \mathrm{Nm}$ amplitude, $1 \mathrm{~Hz}$ sin wave. As shown in the plot below it, the torque tracking error is very small in this case. The next lower plot is the response to a $5 \mathrm{~Hz}$ sin wave input. At $5 \mathrm{~Hz}$, some deterioration in tracking performance was measured as shown on the bottom left hand plot. The peak error was about $1.5 \mathrm{~N}$ or $15 \%$ of the input wave amplitude. The degradation was probably due to the fact that we have ignored servovalve dynamics and valve current limitations.

The right hand plots show the system response using only a standard proportional control law, i.e., $u=K_{p}\left(\tau-\tau_{s}\right)$ with no nonlinear inversion of $\hat{u}$ needed to get $u$. The gain $K_{p}$ was chosen to be the largest value that produced a stable response in the operating range of the cylinder. The lower 
right hand plot shows that the standard proportional controller fails completely due to the large phase lag at $5 \mathrm{~Hz}$.

Given an ideal system, our torque controller drives the torques $\tau(t)$ to any differentiable desired torque $\tau_{s}(t)$. One should then be able to choose the desired torque to be the same as that needed to control a standard torque controlled robot. The following analysis demonstrates how exponential stability of the entire system can be established if one defines $\tau_{s}(t)$ to be an inverse dynamics control law, or to be the control law of Slotine and Li [24].

\section{B. Hierarchical Control}

First we review a result from Vidyasagar [25] on the stability of systems in hierarchical or triangular form. Theorem "Hierarchical System Stability": Consider a system in the form

$$
\begin{aligned}
\dot{x}_{1}(t) & =\boldsymbol{f}_{1}\left(t, \boldsymbol{x}_{1}(t)\right) \\
\dot{\boldsymbol{x}}_{2}(t) & =\boldsymbol{f}_{2}\left(t, \boldsymbol{x}_{1}(t), \boldsymbol{x}_{2}(t)\right) \\
\vdots & \\
\dot{\boldsymbol{x}}_{l}(t) & =\boldsymbol{f}_{l}\left(t, \boldsymbol{x}_{1}(t), \cdots, \boldsymbol{x}_{l}(t)\right)
\end{aligned}
$$

where each $\boldsymbol{x}_{i}(t)$ represents a vector. Suppose for each $i \in$ $\{1, \cdots, l\}$ the following conditions are satisfied:

$$
\begin{gathered}
\boldsymbol{f}_{i} \text { is } C^{1} \\
f_{i}(t, \mathbf{0}, \cdots, \mathbf{0})=\mathbf{0}, \quad \forall t \geq 0
\end{gathered}
$$

and there exists constants $\lambda<\infty$ and $r>0$ such that

$$
\sup _{t \geq 0} \sup _{\overline{\boldsymbol{x}}_{i} \in B_{r}}\left\|\frac{\partial \boldsymbol{f}_{i}\left(t, \overline{\boldsymbol{x}}_{i}\right)}{\partial \overline{\boldsymbol{x}}_{i}}\right\| \leq \lambda
$$

where

$$
\bar{x}_{i}=\left[\begin{array}{c}
x_{1} \\
\vdots \\
x_{i}
\end{array}\right]
$$

Under these conditions, $\overline{\boldsymbol{x}}_{l}=\mathbf{0}$ is a exponentially stable equilibrium of the system (20) if and only if $\boldsymbol{x}_{\boldsymbol{i}}=\mathbf{0}$ is a exponentially stable equilibrium of the systems

$$
\dot{x}_{i}(t)=f_{i}\left(t, \mathbf{0}, \cdots, \mathbf{0}, x_{i}(t)\right)
$$

for each $i \in\{1, \cdots, l\}$. In this theorem, $\|\cdot\|$ is any norm, but $\|\cdot\|_{\infty}$ is the most convenient to use. The notation $B_{r}$ means an open ball of radius $r$.

For our pneumatic robot, the hierarchy consists of the torque control subsystem dynamics, which will be $\boldsymbol{x}_{1}$, and the controlled robot dynamics, which will be $x_{2}$. These two subsystems will be shown to satisfy the criteria for stabilization of a hierarchical system. If we define $\boldsymbol{x}_{1} \equiv d$, then (19) becomes

$$
\dot{\boldsymbol{x}}_{1}=K \boldsymbol{x}_{1}
$$

Because of its simple linear structure, it is clear that it satisfies all the criteria for a subsystem in a hierarchical system.

\section{Inverse Dynamics Hierarchical Control}

One of the most popular control laws for manipulators is inverse dynamics, or computed torque control (see, e.g., Spong and Vidyasagar [26]). In order to use inverse dynamics control for the pneumatic system, assume the robot equations of motion are

$$
\tau=M(q) \ddot{q}+C(q, \dot{q}) \dot{q}+g(q)
$$

and let the desired torque be

$$
\tau_{s}=M(q)\left(K_{p} e+K_{d} \dot{e}+\ddot{q}_{d}\right)+C(q, \dot{q}) \dot{q}+g(q),
$$

where $e=q-q_{d}$. Then with $d=\tau-\tau_{s}$ use the equations of motion, (27) and (28) to obtain

$$
\begin{aligned}
& M(q) \ddot{q}+C(q, \dot{q}) \dot{q}+g(q)-M(q)\left(K_{p} e+K_{d} \dot{e}+\ddot{q}_{d}\right) \\
& -C(q, \dot{q}) \dot{q}-g(q)=d
\end{aligned}
$$

Canceling terms, and using the fact that the inertia matrix is invertible we get

$$
\ddot{e}=K_{p} e+K_{d} \dot{e}+M^{-1}(q) d .
$$

To fit the form of (20), this can be rewritten as

$$
\dot{\boldsymbol{x}}_{2}=\boldsymbol{f}_{2}\left(t, \boldsymbol{x}_{1}(t), \boldsymbol{x}_{2}(t)\right)=\left[\begin{array}{cc}
0 & 1 \\
K_{p} & K_{d}
\end{array}\right] \boldsymbol{x}_{2}+\left[\begin{array}{c}
0 \\
M^{-1}(q)
\end{array}\right] \boldsymbol{x}_{1}
$$

by defining

$$
\boldsymbol{x}_{2} \equiv\left\{\begin{array}{c}
e \\
\dot{e}
\end{array}\right\}
$$

and recalling $\boldsymbol{x}_{1}=d$. Note that because $q=e+q_{d}(t), q$ is a function of $\boldsymbol{x}_{2}$ and time, i.e., $q=q\left(\boldsymbol{x}_{2}, t\right)$, which is in the form required for the theorem.

Clearly $f_{2}$ is $C^{1}$ and its derivatives with respect to any state variable are bounded. Also $f_{2}(t, \mathbf{0}, \mathbf{0})=\mathbf{0}, \forall t \geq 0$. Since $f_{2}\left(t, \mathbf{0}, \boldsymbol{x}_{2}(t)\right)$ is the linear system $\dot{\boldsymbol{x}}_{2}=\left[\begin{array}{cc}0 & 1 \\ K_{p} & K_{d}\end{array}\right] \boldsymbol{x}_{2}, K_{p}$ and $K_{d}$ can easily be chosen to make $\boldsymbol{x}_{2}=\mathbf{0}$ an exponentially stable equilibrium of the system. This subsystem, $\dot{\boldsymbol{x}}_{2}=\boldsymbol{f}_{2}\left(t, \mathbf{0}, \boldsymbol{x}_{2}(t)\right)$, satisfies all the requirements necessary for a subsystem of a hierarchical system. Therefore by the hierarchical system stability theorem, the entire system is exponentially stable.

\section{Slotine Hierarchical Control}

The Slotine and $\mathrm{Li}$ [24] algorithm can also be used to control the robot. For the nonadaptive version, a procedure similar to the above shows that the closed loop system dynamics are

$$
\dot{s}=M^{-1}(q)\left[-C(q, \dot{q}) s-K_{d} s+d\right]
$$

where $s=\dot{e}+\lambda e$.

We can then rewrite the dynamics as (34) and (35), shown at the bottom of the next page. The function $\boldsymbol{f}_{2}$ is continuously differentiable and its derivatives with respect to all state variables are bounded. The criteria of $\boldsymbol{f}_{2}(t, \mathbf{0}, \mathbf{0})=\mathbf{0}$ and $\boldsymbol{x}_{2}=\mathbf{0}$ being an exponentially stable equilibrium of the system $\dot{\mathrm{x}}_{2}=\boldsymbol{f}_{2}\left(t, \mathbf{0}, \boldsymbol{x}_{2}\right)$ are also satisfied, therefore the complete 


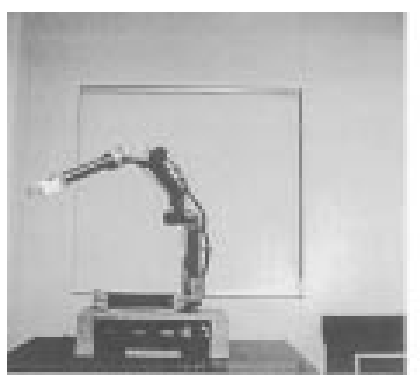

(a)

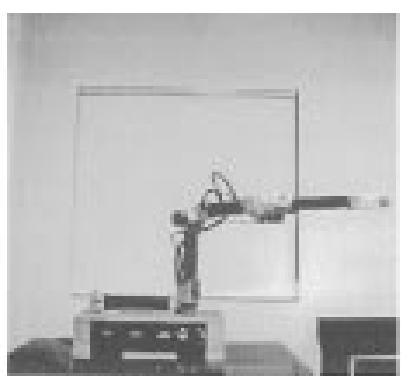

(b)

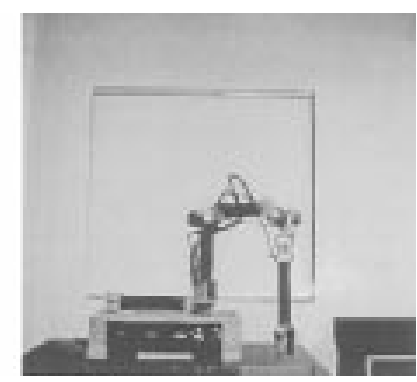

(c)

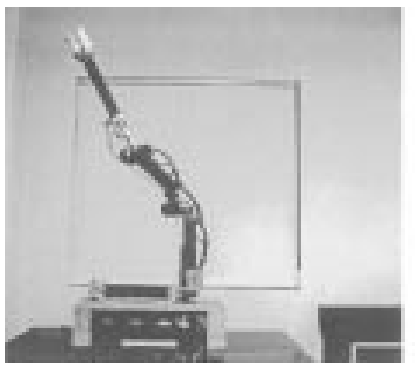

(d)

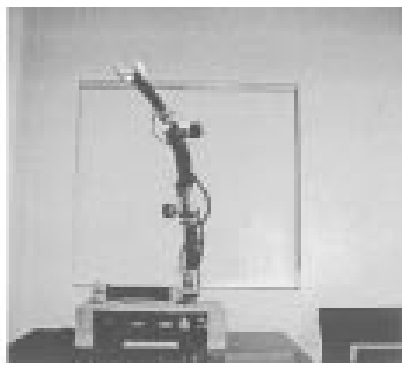

(e)

Fig. 5. Five positions used for testing three degree of freedom algorithms.

system of $\boldsymbol{f}_{1}$ and $\boldsymbol{f}_{2}$ is exponentially stable. The adaptive version of the Slotine and $\mathrm{Li}$ algorithm requires the definition of a third level of the hierarchy to handle the adaptation dynamics.

\section{E. Hybrid Position/Force Control}

As robotic tasks become more advanced, there will be a greater need for robots which don't simply move without contact with the environment. One of the advantages of a pneumatic system is the ability to accurately produce and monitor joint torques based on the cylinder pressures. For this reason, an ideal application for a pneumatic robot is force control. Typically in force control applications a force/torque sensor will be incorporated to close the force control loop. Unfortunately, these sensors are not only costly but fragile. If the sensor could be eliminated while still maintaining good force control, a savings in cost would be achieved with an increase in reliability.

The control law used in the following experiments is a modified version of a hybrid position/force controller given in Lewis et al. [27]. The desired joint torque is defined as

$$
\tau_{s}=M(q) J^{-1}(q)(a-\dot{J}(q) \dot{q})+C(q, \dot{q}) \dot{q}+g(q)+J^{\top}(q) f
$$

where $J(q)$ is the Jacobean matrix defined by $\dot{x}=J(q) \dot{q}$, and $a$ is a desired Cartesian acceleration determined from an outer position loop. After substituting this in to the dynamics of the robot in (27) with the additional $J^{\top}(q) f$ term to represent contact with the environment, the closed loop dynamics with $d=0$ become $\ddot{x}=a$. The task space motion has been globally linearized and decoupled allowing the position and force controllers to be designed independently. For our experiment we will assume position control in the $x$ and $y$ Cartesian directions and force control in the $z$ direction. Let

$$
a=\left\{\begin{array}{l}
a_{x} \\
a_{y} \\
a_{z}
\end{array}\right\}=\left[\begin{array}{ccc}
k_{p} & 0 & 0 \\
0 & k_{p} & 0 \\
0 & 0 & 0
\end{array}\right] e+\left[\begin{array}{ccc}
k_{d} & 0 & 0 \\
0 & k_{d} & 0 \\
0 & 0 & 0
\end{array}\right] \dot{e}
$$

where $e$ is the error in the Cartesian workspace coordinates. This will control the robot motion in the $x y$ plane. The force control normal to the $x y$ plane will be provided through the open loop force term with $f=\left\{\begin{array}{lll}0 & 0 & f_{z}\end{array}\right\}^{\top}$. While many values of $f_{z}$ were tested, in the following experiments $f_{z}$ was set to a $50 \mathrm{~N}$ downward force in one test and to $220 \mathrm{~N}$ in another test. The stability of this control law when combined with the torque control subsystem is similar to the proof of the inverse dynamics controller of Section V-C.

$$
\begin{aligned}
\dot{x}_{2}= & {\left[\begin{array}{cc}
0 & 1 \\
-\lambda+M^{-1}(q)\left[-C(q, \dot{q})-K_{d}\right] \lambda & M^{-1}(q)\left[-C(q, \dot{q})-K_{d}\right]
\end{array}\right] \boldsymbol{x}_{2}+\cdots } \\
& \cdot\left[\begin{array}{c}
0 \\
M^{-1}(q)
\end{array}\right] \boldsymbol{x}_{1} \\
\dot{x}_{2}= & f_{2}\left(t, x_{1}, \boldsymbol{x}_{2}\right)
\end{aligned}
$$




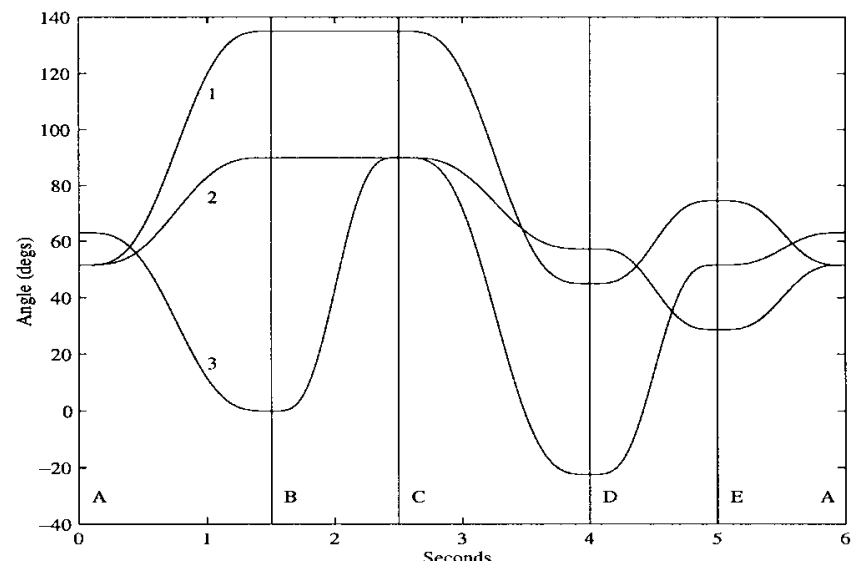

Fig. 6. Trajectory used for testing three degree of freedom algorithms.

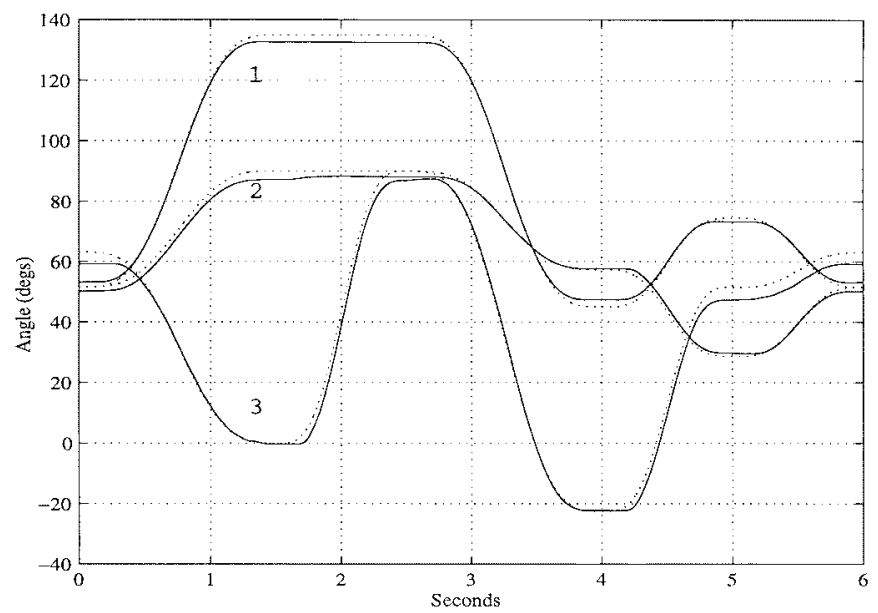

Fig. 7. Tracking the trajectory of Fig. 6 with the hierarchical torque-Slotine controller.

\section{EXPERIMENTS}

Numerous experiments were performed on UCI's three degree of freedom pneumatic robot to test the control algorithms. A trajectory used to test the tracking control law is shown in Fig. 5. The joint space motion that blends the five positions (a)-(e) shown in Fig. 5 is shown in Fig. 6. The positions are labeled in the plot with vertical lines. The individual joint trajectories are also labeled 1,2, and 3 for the base, shoulder, and elbow, respectively.

The hierarchical inverse dynamics control law and the hierarchical Slotine control law were tested under many different conditions including different sampling rates. A representative experimental result is shown in Fig. 7. This plot has the desired trajectory of Fig. 6 shown as a dotted line, and the actual trajectory shown as a solid line. Fig. 8 shows the tracking error in degrees.

The tracking error should theoretically converge to zero, however due to modeling errors such as friction, air flow limitations, and the fact that we have ignored servovalve dynamics, this was not the case. One might wonder how our tracking errors compare to those observed for most electric motor driven robots? This is not easy to answer since the errors are trajectory dependent and there is no standard for comparison between robots. Another consideration is the gear

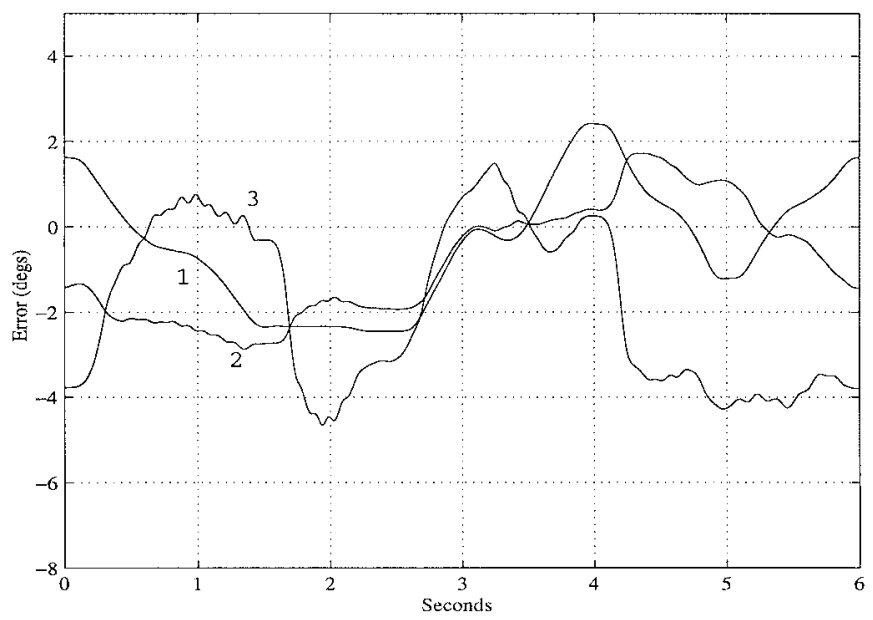

Fig. 8. Error while tracking the above path with the hierarchical torque-Slotine controller.

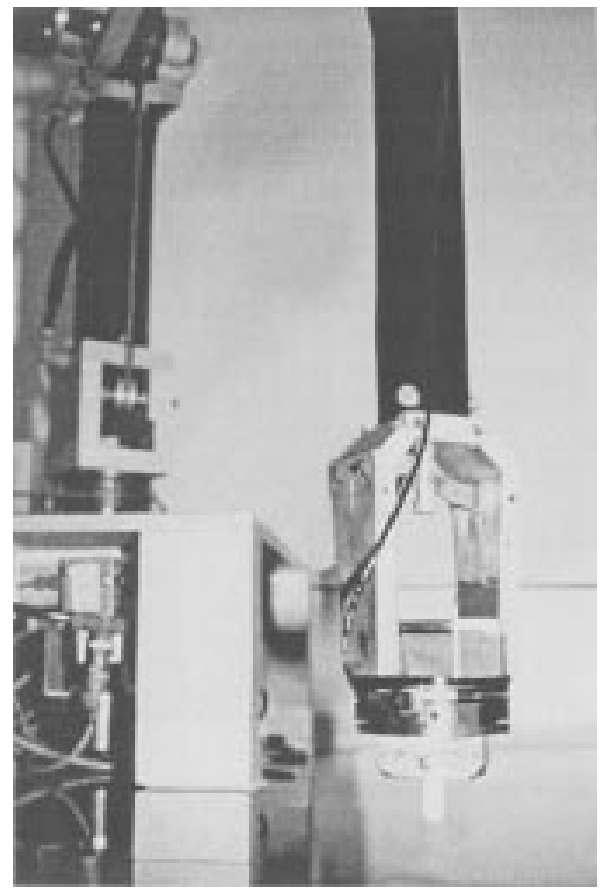

Fig. 9. Force/torque sensor attached to the robot.

reduction used in an electric robot-our robot is a directdrive device and should be compared to these. One of the few experimental studies reported that attempts to quantify tracking error is given in [28]. For the two robots considered in that study and using their best control laws, the root-meansquared errors varied from about $1-3^{\circ}$, which is very close to the errors observed for our pneumatic robot.

\section{A. Tip Force Control}

The tip force control law described in Section V-E was tested on the three degree of freedom robot with a force/torque sensor attached to the tip. Mounted to the sensor was a small fixture for holding a short Teflon rod. The attached sensor is shown in Fig. 9. This sensor was used only to monitor the forces exerted by the robot on the table, its output was not sent to the controller to be used in a feedback loop. 


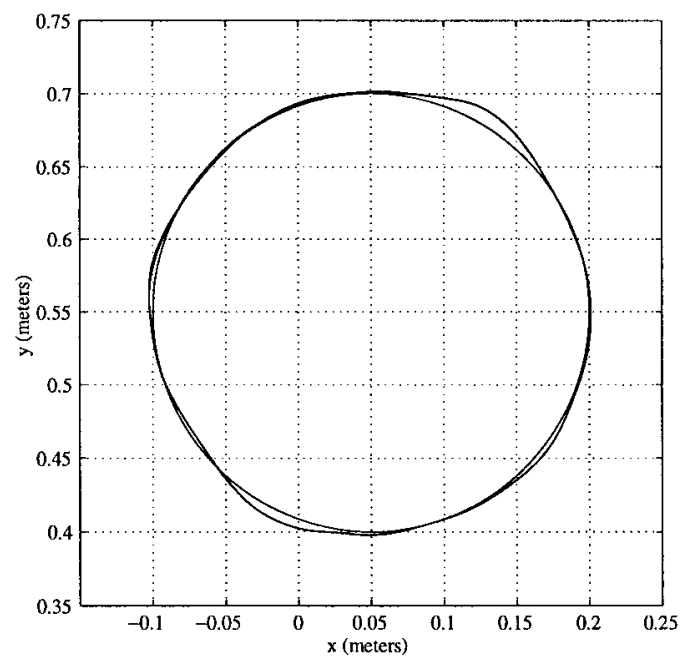

Fig. 10. Position tracking of circle while applying a 50 Newton normal force.

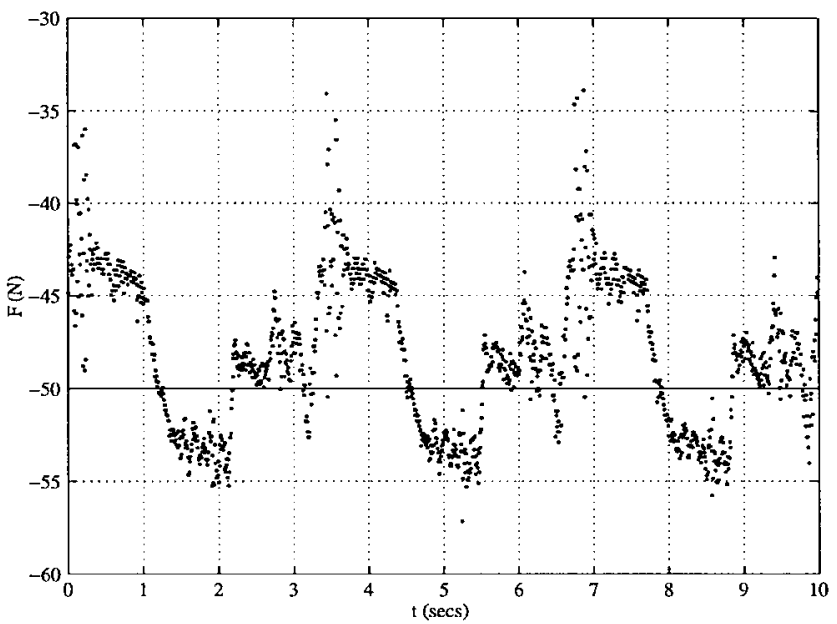

Fig. 11. $Z$ component of tip force from force/torque sensor.

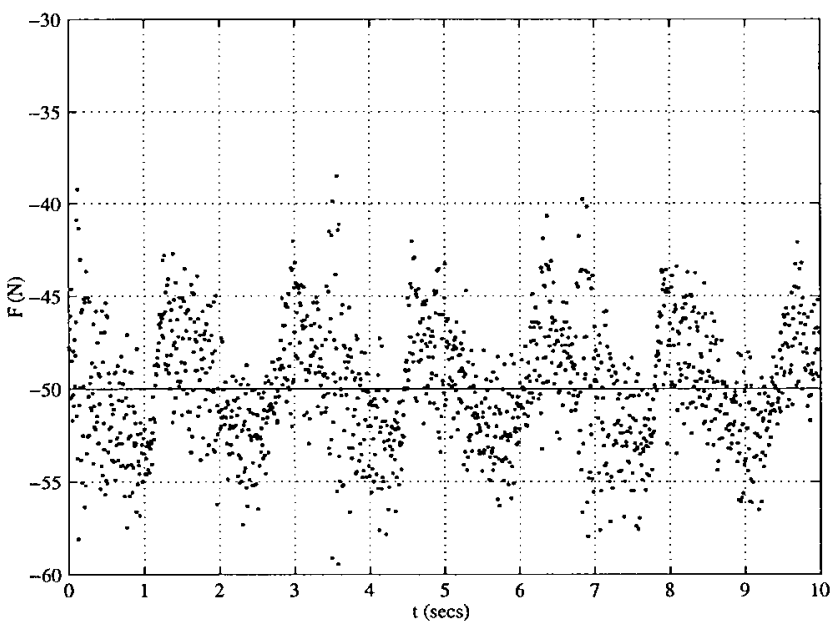

Fig. 12. $Z$ component of tip force from measured joint torques.

Figs. 10-15 show the results of two tests of tracking a circle in the $x y$ plane while applying a 50 Newton $(11 \mathrm{lb})$ and a 220 Newton $(50 \mathrm{lb})$ downward force. The 220 Newton force is just under the maximum possible force given the

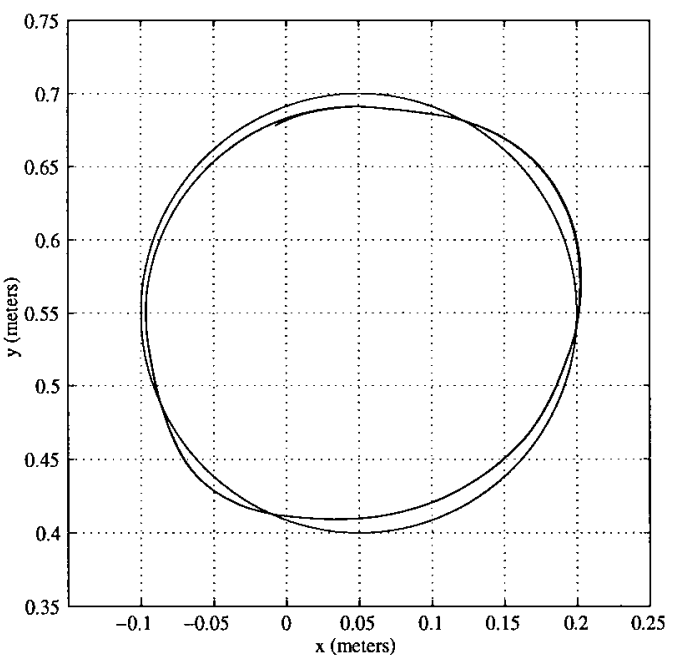

Fig. 13. Position tracking of circle while applying a 220 Newton normal force.

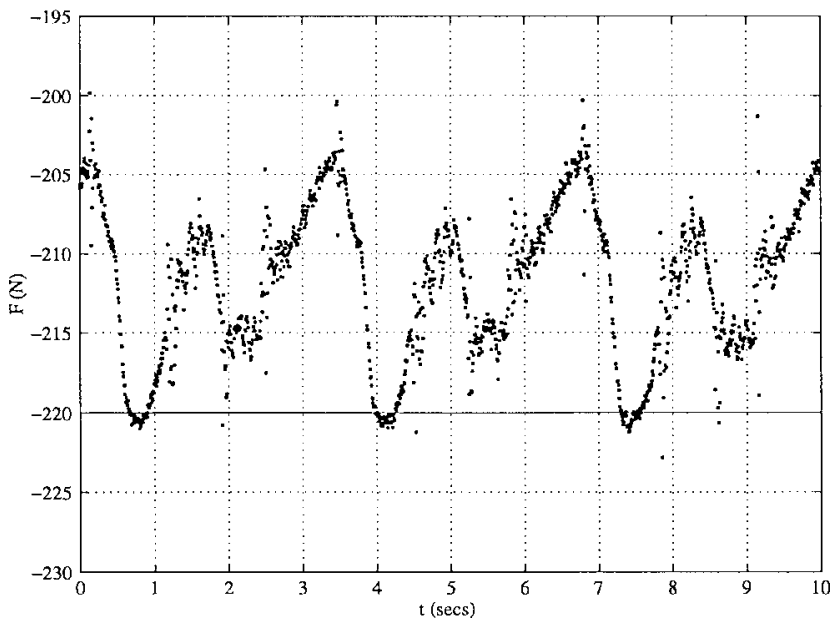

Fig. 14. $Z$ component of tip force from force/torque sensor.

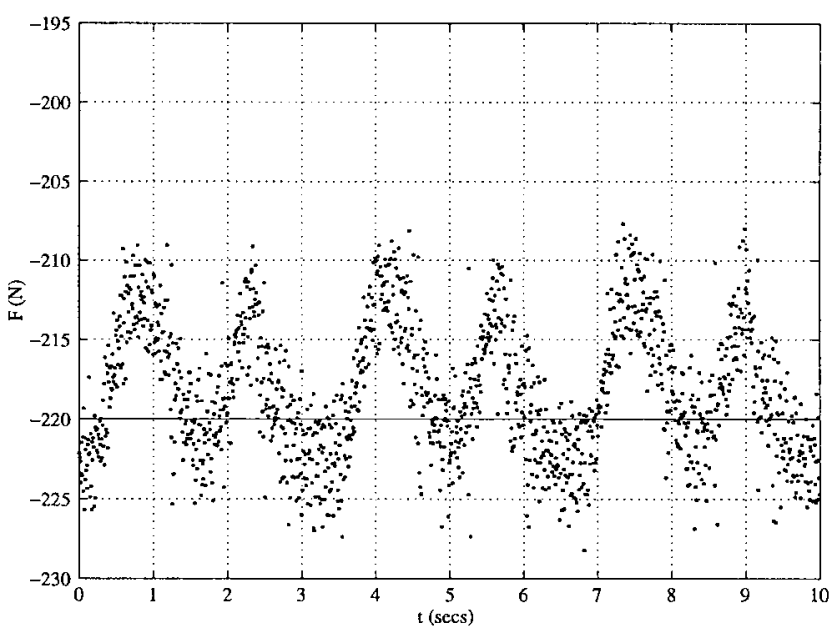

Fig. 15. $Z$ component of tip force from measured joint torques.

robot configuration and the air supply pressure. Shown are the desired and actual Cartesian trajectories, as well as the actual measured tip force from the force-torque sensor (not filtered) and the predicted tip force as computed from the joint torques 
and the dynamic model of the robot. The periodic nature of both plots is due to the fact that the tip of the robot traverses the circle about three times during the test. The fact that the errors repeat suggest that the errors could be removed by careful calibration. Note that in both output force plots even though the force controller is completely open loop, the force is still relatively close to the desired value.

\section{CONCLUSION}

The theory and experiments developed in this research demonstrate that the key to a high performance pneumatic system is a good model. Through system modeling and experimental results, it was found that previous assumptions regarding air flow through a servovalve were not accurate. The experimental data for the air flow through the servovalve did not agree with the predicted flow assumed by previous researchers. This is not surprising due to the complex, three dimensional flows present inside the servovalve. Using the experimental data, a new model was developed which much more closely matched the actual performance of the valve. Experimental tests of the model were performed which revealed that the model did perform well, and could be used to achieve excellent torque tracking at low frequencies $(<5 \mathrm{~Hz})$ with some degradation in torque tracking due to flow saturation occurring at higher frequencies.

Once the valve flow characteristics were determined, a model based control approach was developed that permits the use of most existing control laws for standard torque motor controlled robots. The pneumatic controller is cast into a hierarchical system of torque controller and rigid-body dynamics controller which can be treated as two separate subsystems for stability analysis. The control law tested exhibited good trajectory tracking characteristics for the multiple degree of freedom robot used in the experiments.

One of the most promising capabilities of the pneumatically actuated robot system is its ability to produce tip forces from the measured joint torques. Using only open loop force control in conjunction with a controller for tracking motion tangential to a surface, the robot was able to produce a tip force without the use of a force/torque sensor to close the loop. This was performed on a rigid surface without a compliant end effector. In contrast to some previous experimental research, no instabilities in tip force control were encountered. Hence, the natural compliance of the compressed air is advantageous for force control applications. The experimental results achieved in this research demonstrate the remarkable potential of pneumatically actuated robot systems.

\section{APPENDIX}

\section{LiST OF Symbols RELATED TO THE ACUATATOR}

$\begin{array}{ll}\theta & \text { Angular position of the pulley. } \\ r & \text { Radius of the cable pulley. } \\ l & \text { Length of cylinder stroke. } \\ A_{c} & \text { Cylinder cross sectional area. } \\ A_{o} & \text { Effective valve orifice area. } \\ \text { A, B } & \text { Reference to sides of cylinder. }\end{array}$
$\dot{m}_{a}, \dot{m}_{b}$
Mass flow rate of air into chambers $\mathrm{A}$ and
$T_{s}$
$p_{a}, p_{b}$ B.
$p_{\text {sup }}, p_{\text {exh }}$
$v_{a}, v_{b}$
$v_{a, \text { end }}, v_{b, \text { end }}$
Air supply temperature.
$c_{v}, c_{p}$
Absolute pressures in chambers A and B.
Supply and exhaust pressures.
Volume of chambers A and B.
Volumes not swept by piston in chambers
$\mathrm{A}$ and $\mathrm{B}$.
$c_{v}, c_{p} \quad$ Constant volur
$k=c_{p} / c_{v} \quad$ For air this ratio is 1.4
$\dot{Q} \quad$ Rate of heat transfer to the cylinder.
$R \quad$ Universal gas constant.
$\tau \quad$ Joint torque.
$u \quad$ Servovalve input current.
$c_{f 1}, c_{f 2}, c_{e 1}, c_{e 2}$ Valve flow constants for fill and exhaust.

\section{REFERENCES}

[1] J. L. Shearer, "Study of pneumatic processes in the continuous control of motion with compressed air-i, ii," Trans. ASME, pp. 233-242, Feb. 1956.

[2] C. R. Burrows and C. R. Webb, "Use of root loci in design of pneumatic servo motors," Control, pp. 423-427, Aug. 1966.

[3] D. R. Vaughan, "Hot-gas actuators: Some limits on the response speed," ASME J. Basic Eng., pp. 113-119, Mar. 1965.

[4] S. Liu and J. E. Burrows, "An analysis of a pneumatic servo sustem and its application to a computer-controlled robot," ASME J. Dynam. Syst., Meas., Contr., vol. 115, no. 3, pp. 427-433, Sept. 1988.

[5] J. Pu and R. H. Weston, "A new generation of pneumatic servos for industrial roots," Robotica, vol. 7, pp. 17-23, Jan. 1989.

[6] B. W. McDonell and J. E. Bobrow, "Adaptive tracking control of an air powered robot actuator," ASME J. Dynam. Syst., Meas., Contr., vol. 115, no. , pp. 427-433, Sept. 1993.

[7] J. Y. Lai, C. H. Meng, and R. Singh, "Accurate position control of a pneumatic actuator," ASME J. Dynam. Syst., Meas., Contr., pp. 734-739, Dec. 1990

[8] J. Tang and G. Walker, "Variable structure control of a pneumatic actuator," ASME J. Dynam. Syst., Meas., Contr., vol. 117, no. 1, pp. 88-92, Mar. 1995.

[9] A. K. Paul, J. K. Mishra, and M. G. Radke, "Reduced order sliding mode control for pneumatic actuator," IEEE Trans. Contr. Syst. Technol., vol. 2, pp. 271-276, Sept. 1994.

[10] C. Kunt and R. Singh, "A linear time varying model for on-off valve controlled pneumatic actuators," ASME J. Dynam. Syst., Meas., Contr., pp. 740-747, Dec. 1990.

[11] A. Bouhal, E. Richard, and S. Scavarda, "An experimental comparative study of linear and nonlinear adaptive pressure regulation," in Proc. 6th Bath Int. Fluid Power Workshop Model. Simulat., 1993, pp. 225-238.

[12] T. Kimura, S. Hara, T. Fujita, and T. Kagawa, "Control for pneumatic actuator systems using feedback linearization with disturbance rejection," in Proc. 1995 Amer. Contr. Conf., Seattle, WA, June 1995, pp. 825-829.

[13] S. Kawmura, K. Miyata, H. Hanafusa, and K. Isisa, "Pi type hierarchical control scheme for pneuamtic robots," in Proc. 1989 IEEE Int. Conf. Robot. Automat., Scottsdale, AZ, May 1989, pp. 1953-1858.

[14] S. C. Jacobsen, J. E. Wood, D. E. Knutti, and K. B. Biggers, "The utah/mit dextrous hand: Work in progress," Int. J. Robot. Res., vol. 3, no. 4, pp. 21-50, 1984.

[15] P. D. Henri and J. M. Hollerbach, "An analytical and experimental investigation of a jet pipe controlled electropneumatic actuator," in Proc. IEEE Int. Conf. Robot. Automat., San Diego, CA, May 1994, pp. 300-306.

[16] D. Ben-Dov and S. E. Salcudean, "A force-controlled pneumatic actuator," IEEE Trans. Robot. Automat., vol. 11, pp. 906-911, Dec. 1995.

[17] G. H. Pfruendschuh, V. Kumar, and T. G. Sugar, "Design and control of a 3 dof in-parallel actuated maniplator," in Proc. Int. Conf. Robot. Automat., Sacramento, CA, 1991, pp. 1659-1664.

[18] G. S. Chirikjian and I. Ebert-Uphoff, "Efficient workspace generation for binary manipulators with many actuators," J. Robot. Syst., vol. 12, no. 6, pp. 383-400, June 1995.

[19] R. Atchley, Servovalve Model 211APN, Typical Performance Specifications. Los Angeles, CA: Atchley Controls. 
[20] Y. Kawakami, J. Akao, S. Kawai, and T. Machiyama, "Some considerations on the dynamic characteristics of pneumatic cylinders," J. Fluid Contr., vol. 19, no. 2, pp. 22-36, Sept. 1988.

[21] R. L. Daugherty, J. B. Franzini, and E. J. Finnemore, Fluid Mechanics with Engineering Applications. New York: McGraw-Hill, 1985.

[22] B. W. McDonell, Modeling, Identification, and Control of a Pneumatically Actuated Robotic Manipulator, Ph.D. dissertation, Dept. Mech. \& Aerosp. Eng., Univ. California, Irvine, 1996.

[23] P. V. Kokotovic, "The joy of feedback: Nonlinear and adaptive," IEEE Contr. Syst. Mag., pp. 7-17, June 1992.

[24] J.-J. E. Slotine and W. Li, Applied Nonlinear Control. Englewood Cliffs, NJ: Prentice-Hall, 1991.

[25] M. Vidyasagar, Nonlinear Systems Analysis, 2nd ed. Englewood Cliffs, NJ: Prentice-Hall, 1993.

[26] M. W. Spong and M. Vidyasagar, Robot Dynamics and Control. New York: Wiley, 1989.

[27] F. L. Lewis, C. T. Abdallah, and D. M. Dawson, Control of Robot Manipulators. New York: MacMillan, 1993.

[28] L. L. Whitcomb, A. A. Rizzi, and D. E. Koditschek, "Comparative experiments with a new adaptive controller for robot arms," IEEE Trans. Robot. Automat., vol. 9, pp. 59-70, Feb. 1993.

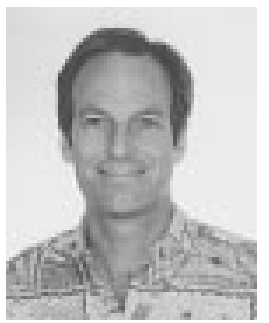

James E. Bobrow received the M.S. and Ph.D. degrees in engineering from the Mechanics and Structures Department, University of California, Los Angeles, in 1979 and 1983, respectively.

$\mathrm{He}$ is a Professor of Mechanical and Aerospace Engineering at the University of California, Irvine (UCI). After graduate school, he was a Senior Programmer Analyst with McDonnell Douglas Automation Company, where he developed CAM software for the Unigraphics system. In June 1984, he joined UCI as an Assistant Professor, where he conducted research in robotics and applied control systems. From 1991 to 1992 , he was a Visiting Associate Professor in the Computer Science Department, Stanford University, Stanford, CA, where he investigated applications of numerical optimization algorithms to learning systems. He has published over 70 research papers in robotics, applied control systems, numerical optimization techniques, and computer-aided design.

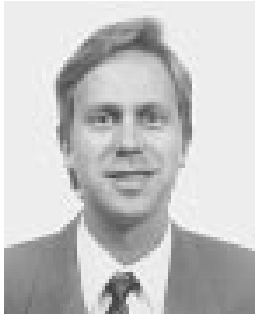

Brian W. McDonell received the B.S. and Ph.D. degrees in mechanical engineering from the University of California, Irvine, in 1989 and 1996, respectively.

His research interests are in the area of computer controlled mechanical systems. He has designed novel controllers for electrically, pneumatically, and hydraulically actuated systems as well as for internal combustion engines. He is currently working at ATL Products, Irvine, CA, a manufacturer of automated computer data storage products. 